\title{
Successful use of a covered, retrievable stent to seal a ruptured mucosal flap safety valve during peroral endoscopic myotomy in a child with achalasia
}

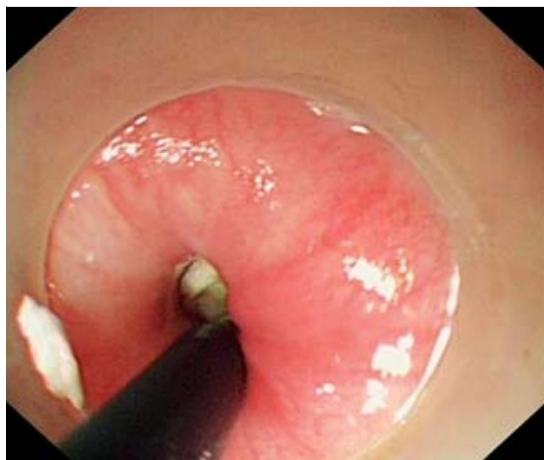

Fig. 1 Prior to carrying out peroral endoscopic myotomy (POEM) in an 8-year-old child with dysphagia, the cardia was too tight for an endoscope to pass through.

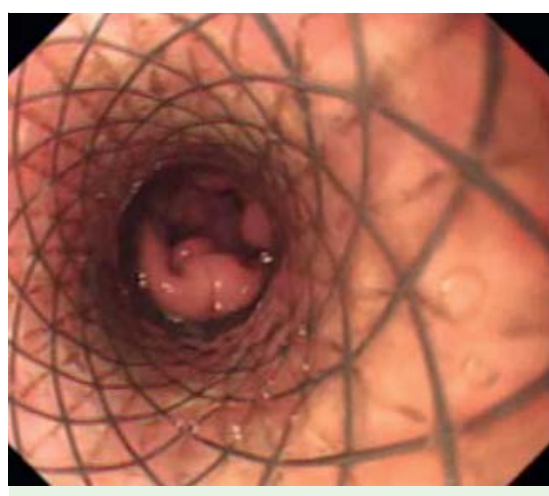

Fig. 4 A covered, retrievable Ni-Ti stent was used to seal the rupture.

An 8-year-old boy was admitted to our hospital due to intermittent episodes of dysphagia over the past 5 years, which was severely affecting his physical growth and quality of life. He was diagnosed as having achalasia 3 years ago, and had twice undergone endoscopic balloon dilation. However, both times the dysphagia recurred very soon after the dilation procedure. The child was only able to swallow fluids at the time of admission.

We carried out peroral endoscopic myotomy (POEM) after careful evaluation. Prior to the procedure, the cardia was so tight that the endoscope could not pass through ( $\bullet$ Fig. 1). A submucosal injection was commenced at the level of the midesophagus, approximately $8 \mathrm{~cm}$ proximal

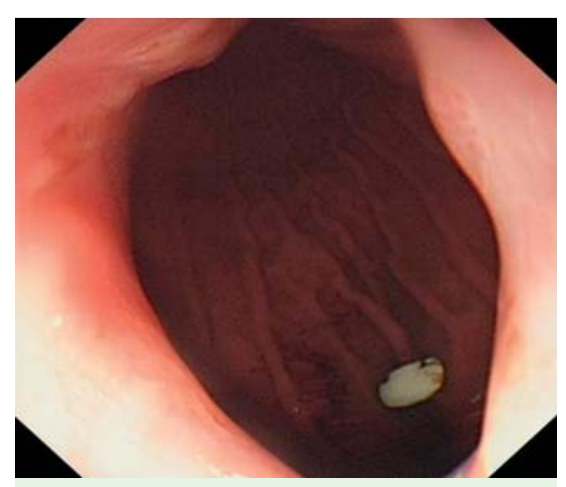

Fig. 2 The opened cardia after peroral endoscopic myotomy (POEM).

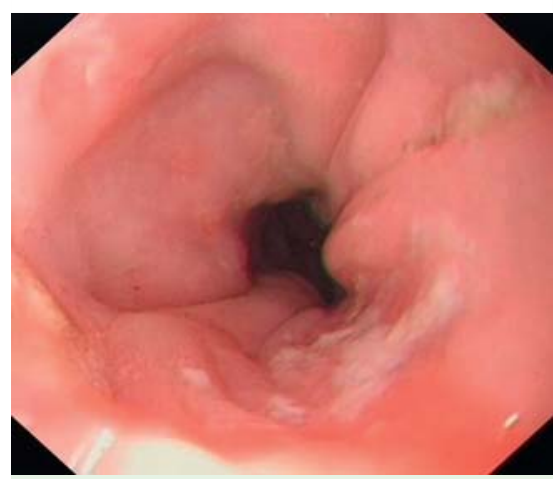

Fig. 5 After 3 weeks, the stent was removed and the rupture of mucosal flap healed well.

to the cardia, and a submucosal tunnel was created accordingly. The muscle layer was completely divided starting $2 \mathrm{~cm}$ distal to the mucosal entry and ending within the stomach $3 \mathrm{~cm}$ distal to the cardia. After the myotomy, the endoscope advanced smoothly through the cardia ( $\bullet$ Fig.2). However, the submucosal scarring resulting from the previous balloon dilation made the procedure very difficult, and a 2-cm rupture of the mucosal flap safety valve occurred at the lower esophagus ( Fig.3). Due to the risk of restenosis, we did not close the rupture with endoscopic clips. Instead, a covered, retrievable nickel-titanium (Ni-Ti) stent was placed to seal the rupture ( Fig.4). The stent was removed after 3 weeks when the rupture had healed ( $\bullet$ Fig.5).

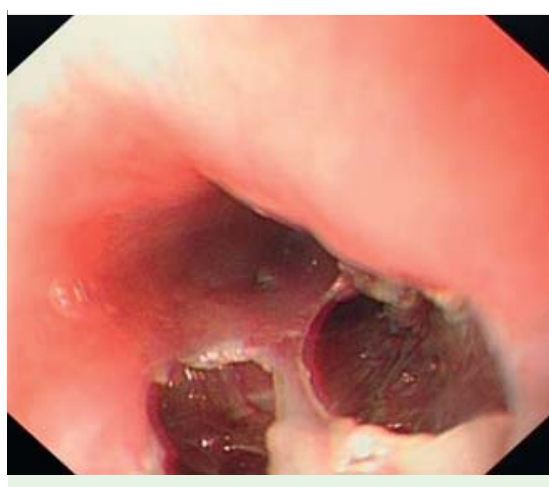

Fig. 3 The mucosal flap safety valve ruptured in an irregular fashion at the level of the lower esophagus.

The child was easily able to eat solid food 3 days after POEM. At follow-up 1 month after POEM, the child's weight had increased by $5 \mathrm{~kg}$, and the barium meal traveled through the cardia without delay ( $\bullet$ Fig.6). The pressure at the lower esophageal sphincter decreased from $90 \mathrm{mmHg}$ before POEM to $16 \mathrm{mmHg}$.

POEM is a novel endoscopic esophagomyotomy procedure for the treatment of achalasia and was first reported by Inoue et al. [1] in 2010. Although several studies with favorable results have been published [2-5], the complications related to the procedure and their management are still not fully elucidated. As far as we are aware, this is the first report of use of a covered, retrievable Ni-Ti stent to seal a ruptured mucosal flap safety valve during POEM.

Endoscopy_UCTN_Code_CPL_1AH_2AK

Competing interests: None

\section{T. Ling ${ }^{1}$, Q. Pei ${ }^{1,2}$, J. Pan ${ }^{1}$, X. Zhang1, Y. Lv ${ }^{1}$, W. Li ${ }^{1}, X$. Zou $^{1}$}

${ }^{1}$ Department of Gastroenterology, Affiliated Drum Tower Hospital of Nanjing University, Medical School, Nanjing, China

2 Department of Gastroenterology, Renmin Hospital of Wuhan University, Wuhan, China 


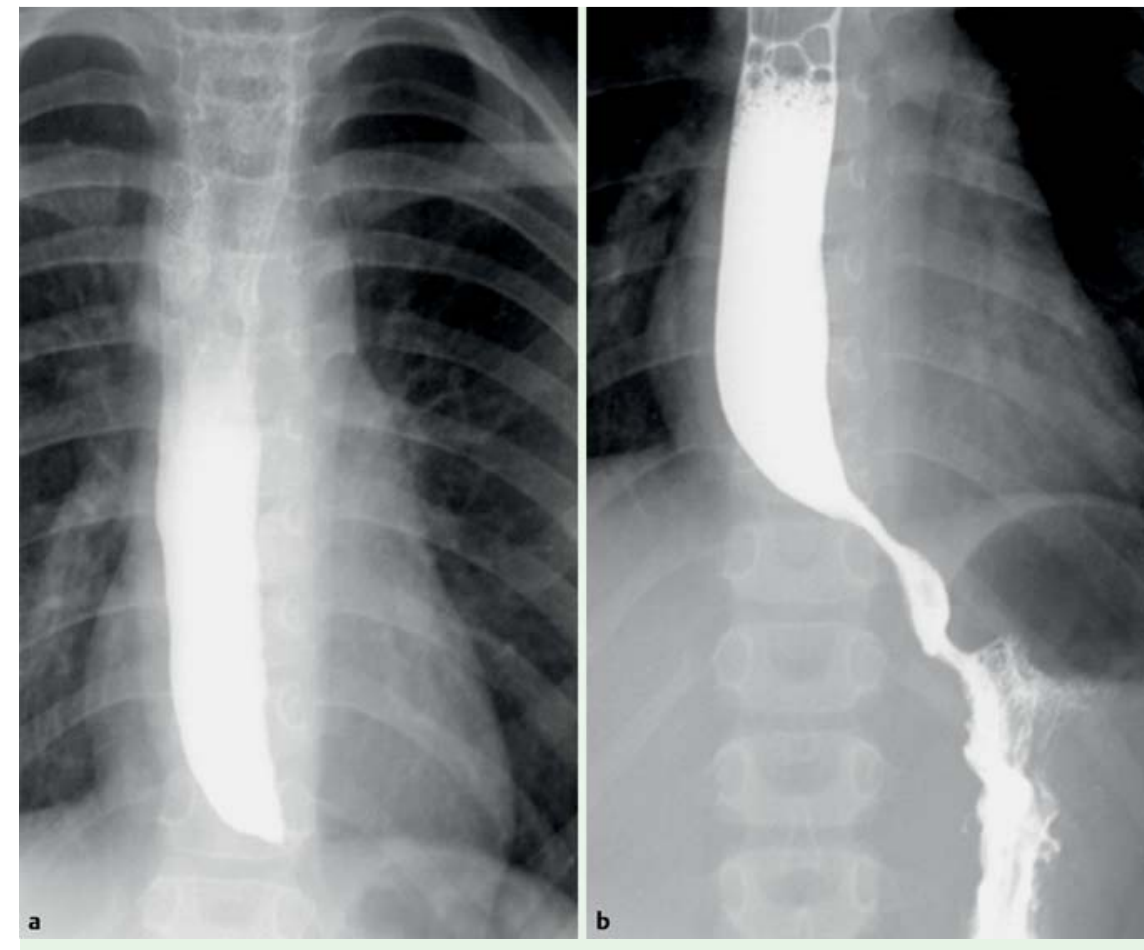

Fig. 6 a The narrow and beak-shaped cardia on barium swallow before peroral endoscopic myotomy (POEM). b The barium meal easily passed through the cardia 1 month after POEM.

\section{References}

1 Inoue H, Minami H, Kobayashi Yet al. Peroral endoscopic myotomy (POEM) for esophageal achalasia. Endoscopy 2010; 42: $265-$ 271

2 Swanstrom LL, Rieder E, Dunst CM. A stepwise approach and early clinical experience in peroral endoscopic myotomy for the treatment of achalasia and esophageal motility disorders. J Am Coll Surg 2011; 213: $751-756$

3 Von Renteln D, Inoue H, Minami $\mathrm{H}$ et al. Peroral endoscopic myotomy for the treatment of achalasia: a prospective single center study. Am J Gastroenterol 2012; 107: 411 417

4 Costamagna G, Marchese M, Familiari P et al. Peroral endoscopic myotomy (POEM) for oesophageal achalasia: Preliminary results in humans. Dig Liver Dis 2012; 44: 827-832

5 Ren Z, Zhong Y, Zhou $P$ et al. Perioperative management and treatment for complications during and after peroral endoscopic myotomy (POEM) for esophageal achalasia (EA) (data from 119 cases). Surg Endosc 2012, [Epub ahead of print]

\section{Bibliography}

DOI http://dx.doi.org/

10.1055/s-0032-1325977

Endoscopy 2013; 45: E63-E64

(c) Georg Thieme Verlag KG

Stuttgart · New York

ISSN 0013-726X

\section{Corresponding author}

\section{Zou}

Department of Gastroenterology

Affiliated Drum Tower Hospital of Nanjing

University Medical School

Nanjing, 210008

China

zouxiaoping795@hotmail.com 